\title{
Probing anomalous $t q h$ couplings via single top production in associated with the Higgs boson at the HE-LHC and FCC-hh
}

\author{
Yan-Ju Zhang ${ }^{\mathrm{a}}$, Jie-Fen Shen ${ }^{\mathrm{b}}$ \\ School of Biomedical Engineering, Xinxiang Medical University, Xinxiang 453003, People's Republic of China
}

Received: 28 June 2020 / Accepted: 17 August 2020 / Published online: 2 September 2020

(C) The Author(s) 2020

\begin{abstract}
We investigate the prospects for discovering the Flavour Changing Neutral Current (FCNC) $t q h$ couplings via the process $p p \rightarrow t h$ at the proposed High Energy Large Hadron Collider (HE-LHC) and Future Circular Collider in hadron-hadron mode (FCC-hh) including the realistic detector effects. The relevant SM backgrounds are considered in the cut based analysis to obtain the limits on the the branching ratios of $t \rightarrow q h(q=u, c)$, followed by the leptonic decay channel of the top quark and diphoton decay channel of the Higgs boson. The upper limits on the FCNC branching ratios at $95 \%$ confidence level (CL) and the $5 \sigma$ discovery reach for the different integrated luminosities are obtained. It is shown that at the $27 \mathrm{TeV}$ HE-LHC with an integrated luminosity of $15 \mathrm{ab}^{-1}$ and at the $100 \mathrm{TeV}$ FCC-hh with an integrated luminosity of $30 \mathrm{ab}^{-1}$, the $\mathrm{BR}(t \rightarrow u h)(\mathrm{BR}(t \rightarrow c h))$ can be probed, respectively, to $4.4(6.4) \times 10^{-5}$ and $1.3(1.6) \times 10^{-5}$ at the $95 \%$ CL.
\end{abstract}

\section{Introduction}

The discovery of the Higgs boson by the Large Hadron Collider (LHC) $[1,2]$ experiments has opened up a new era of the direct searches for new physics (NP) beyond the Standard Model (SM). One of the possible anomalous interactions is the flavour-changing neutral currents (FCNC) process mediated by the Higgs boson, and such processes are forbidden in $\mathrm{SM}$ at tree level and are strongly suppressed at loop-level due to the Glashow-Iliopoulos-Maiani (GIM) mechanism [3,4]. Being the most massive elementary particle in the SM, the top quark is an excellent probe not only to search the dynamics of electroweak symmetry breaking, but also to test SM and NP beyond the SM. The flavor-violating Higgs couplings involving a top quark in the SM, i.e., the vertex $\operatorname{tqh}(q=u, c)$,

\footnotetext{
a e-mail: yanjuzhang@xxmu.edu.cn

be-mail: shjf@xxmu.edu.cn (corresponding author)
}

are out of range for current LHC experimental sensitivities. While in some NP scenarios such as supersymmetry [5-7], two-Higgs-doublet model [8-12], 3-3-1 model [13], and other NP models by extending the Higgs sector [14,15], the branching ratios of top quark FCNC decays $t \rightarrow q h$ are predicted promisingly at the order of $10^{-6}-10^{-3}$. Thus searches for such FCNC processes are very important and could be considered as a clear signal for NP beyond the SM [16-18].

By searching for $p p \rightarrow t \bar{t}$ process with one top decaying to $W b$ and the other assumed to decay to $h q$, the ATLAS and CMS collaborations have given the upper limits on the top quark FCNC branching ratios at $95 \%$ Confidence Level (CL) at $\sqrt{s}=13 \mathrm{TeV}$ [19-22]. For example, the combination of these searches with ATLAS yields observed limits [21]: $\operatorname{Br}(t \rightarrow u h) \leq 1.2 \times 10^{-3}$, and $\mathrm{Br}(t \rightarrow c h) \leq 1.1 \times 10^{-3}$. As a more promising and realistic project, the future HighLuminosity LHC (HL-LHC) expects to reach branching $B r(t \rightarrow u h) \leq 2.4 \times 10^{-4}$ and $\operatorname{Br}(t \rightarrow c h) \leq 1.5 \times 10^{-4}$ with integrated luminosity of $3000 \mathrm{fb}^{-1}$ at $\sqrt{s}=14 \mathrm{TeV}$ with a full simulation of the ATLAS detector upgrade $[23,24]$.

At present there is no any experimental evidence of the FCNC process. One can expect that future research and improvements on these bounds with the proposed higher center-of-mass energy colliders [25], such as High Energy LHC (HE-LHC) with $27 \mathrm{TeV}$ c.m. energy [26] and Future Circular Collider in hadron-hadron mode (FCC-hh) with 100 TeV c.m. energy [27]. Very recently, the authors of Ref. [28] investigated the $t h j$ final signal by considering $t h q$ FCNC couplings from three different subprocesses at the HE-LHC and FCC-hh. Their results show that the top pair production process with one of top FCNC decay could give dominant contributions. The aim of this work is to investigate the limits on the anomalous $t q h$ couplings by considering the process $p p \rightarrow t h$ at the HE-LHC and FCC-hh projects with the decay chains: $t \rightarrow W^{+} b \rightarrow \ell^{+} \nu b$ and $h \rightarrow \gamma \gamma$. Realistic detector effects are included in the production of signal and 
background processes. The sensitivity of anomalous FCNC $t q h$ couplings are searched by using multivariate analysis. Finally, the results are reported respectively for HE-LHC and FCC-hh and compared with other phenomenological predictions.

This paper is arranged as follows. In Sect. 2, the cross section of the the signal process $p p \rightarrow t h$ is calculated at the HE-LHC and FCC-hh. This section also includes the estimations for the signal and background, the simulations and the detector effects for HE-LHC and FCC-hh. We also give the 95\% confidence level limits of HE-LHC and FCC-hh and compare with the LHC measured limits. Furthermore, we also consider the effects of the systematic uncertainty on the backgrounds. Finally, we conclude and summarize our main results and findings in Sect. 3.

\section{Analysis strategy and numerical result}

In general, the relevant top quark FCNC couplings is explored in a model-independent way considering the most general effective Lagrangian approach [4]. The Lagrangian of the FCNC $t q h$ interactions can be written as

$$
\mathcal{L}=\kappa_{t u h} \bar{t} H u+\kappa_{t c h} \bar{t} H c+h . c .,
$$

where the parameters $\kappa_{t u h}$ and $\kappa_{t c h}$ represent the strength of $t q h$ FCNC interactions. Such FCNC interactions have been phenomenologically studied in many final states with various sensitivities [29-37].

With the above effective interactions, the Feynman diagrams for the production of single top quark associated with the Higgs boson are shown in Fig. 1. Obviously, the conjugate process $p p \rightarrow \bar{t} h$ production can also occur at the tree level which do not included in Fig. 1. For the simulations of the HE-LHC and FCC-hh collider phenomenology, we first use the FeynRules [38] to generate the Universal FeynRules Output (UFO) files [39]. The leading order cross sections are obtained by using MadGraph5-aMC@NLO [40] with NNPDF23L01 parton distribution functions (PDFs) [41] considering the renormalization and factorization scales to be $\mu_{R}=\mu_{F}=\mu_{0} / 2=\left(m_{t}+m_{h}\right) / 2$. We take $\kappa_{t q h} \leq 0.04$ to satisfy the upper limit from the current LHC experiments

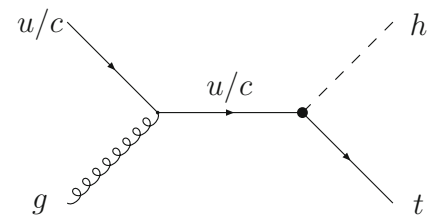

(a)

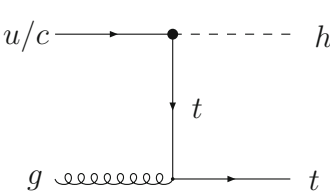

(b)
Fig. 1 The Feynman diagrams for $t h$ production at the LHC through FCNC tqh interactions
Table 1 The cross sections (in fb) of the subprocess $p p \rightarrow t h$ with $\kappa_{t q h}=0.01$ at HE-LHC and FCC-hh

\begin{tabular}{lll}
\hline Process & HE-LHC $(27 \mathrm{TeV})$ & FCC-hh $(100 \mathrm{TeV})$ \\
\hline$u g \rightarrow t h$ & 83.3 & 459.6 \\
$\bar{u} g \rightarrow \bar{t} h$ & 16.4 & 138.6 \\
$g(c+\bar{c}) \rightarrow h(t+\bar{t})$ & 18.6 & 172.5 \\
\hline
\end{tabular}

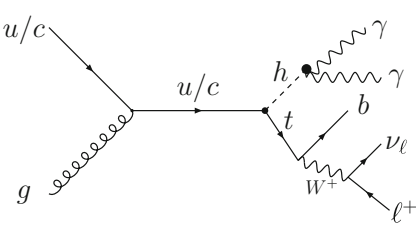

(a)

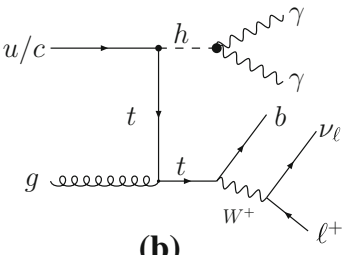

(b)
Fig. 2 The Feynman diagram for the $p p \rightarrow t h$ process including the leptonic decay of top quark and diphoton decay of the Higgs boson

[28]. The numerical values of the input parameters are taken as follows [42]:

$$
\begin{aligned}
m_{h} & =125.1 \mathrm{GeV}, \quad m_{t}=172.9 \mathrm{GeV}, \quad m_{W}=80.379 \mathrm{GeV}, \\
m_{Z} & =91.1876 \mathrm{GeV}, \quad \alpha_{s}\left(m_{Z}\right)=0.1185, \\
G_{F} & =1.166370 \times 10^{-5} \mathrm{GeV}^{-2} .
\end{aligned}
$$

The numerical values of the cross sections are shown in Table 1 at the leading order. One can see that the cross section of $\bar{u} g \rightarrow \bar{t} h$ is overwhelmed by $u g \rightarrow t h$ due to the difference between the $u$-quark and $\bar{u}$-quark PDF of the proton. Thus, if we consider the leptonic top decay modes, more leptons will be observed than anti-leptons for a given centerof-mass (c.m.) energy and luminosity. However, since the $c$-quark and $\bar{c}$-quark have the similar small PDF, the cross section of $\bar{c} g \rightarrow \bar{t} h$ is same to that of $c g \rightarrow t h$ and they are much smaller than the cross section of $u g \rightarrow t h$ for the same values of $\kappa_{t q h}$. This implies that the sensitivity to the FCNC coupling parameter $\kappa_{t u h}$ will be better than $\kappa_{t c h}$.

\subsection{Monte Carlo simulations}

The signal is mainly produced through the process

$p p \rightarrow t\left(\rightarrow W^{+} b \rightarrow \ell^{+} \nu b\right) h(\rightarrow \gamma \gamma)$,

where $\ell=e, \mu$. Note that the analysis has included the charge-conjugate process. The final state for the signal is characterised by two photons, one lepton, one b-jet and missing transverse energy from the undetected neutrino, as shown in Fig. 2. Certainly, the $p p \rightarrow t \bar{t} \rightarrow t h j$ process should also be a source of the signal if the final light quark is missed by the detector. Due to small production cross sections for $c(\bar{c}) \rightarrow h t(\bar{t})$ processes, this additional contribution is very 
significant for detecting the $\lambda_{t c h}$ couplings [29]. Therefore, we also include the $p p \rightarrow t \bar{t} \rightarrow t h j$ process and focus on the final topology from the $p p \rightarrow t h$ process without the extra light jet. Considering the signal scenarios, the following relevant background processes which have similar final state need to be taken into account: the resonant backgrounds $t \bar{t} h$ and $t h j$ in which the Higgs boson decays to diphotons, and the non-resonant backgrounds $t \bar{t} \gamma \gamma, t j \gamma \gamma$ and $\gamma \gamma W^{ \pm} j j$. We do not consider processes where the light jet is mis-tagged as the photon, which is beyond the scope of this paper.

The signal and background samples are generated at leading order (LO) by using the Monte Carlo event generator MadGraph5-aMC@NLO [40], interfaced to Pythia 8.20 [43] for the parton showering. All produced jets are forced to be clustered using the FASTJET 3.2 [44] assuming the anti- $k_{t}$ algorithm with a cone radius of $R=0.4$ [45]. All event samples are fed into the Delphes 3.4.2 [46] with the default HE-LHC and FCC-hh detector card. Finally, event analysis is performed by using MadAnalysis5 [47]. Here it should be mentioned that we rescale the leading order cross sections to the corresponding higher order QCD results with the $K$-factor, i.e., $K=1.5$ for the $p p \rightarrow t h$ process $[29,48]$, $K=1.4$ for the $p p \rightarrow t \bar{t} \rightarrow t h j$ process [49] and $K=1.3$ for the $p p \rightarrow t \bar{t} h$ process [49-51]. For the sake of simplicity, others SM background processes, we have rescaled their cross sections by a $K$-factor of 1.5 . This approximation does not have a significant impact on our derived sensitivities.

In order to identify objects, we chose the basic cuts at parton level for the signals and SM backgrounds as follows:

$$
\begin{array}{rlll}
\mathrm{HE}-\mathrm{LHC}: & p_{T}^{\ell / \gamma}>25 \mathrm{GeV}, & p_{T}^{j / b}>30 \mathrm{GeV}, & \left|\eta_{i}\right|<2.5, \\
\mathrm{FCC}-\mathrm{hh}: & p_{T}^{\ell / \gamma}>25 \mathrm{GeV}, & p_{T}^{j / b}>30 \mathrm{GeV}, & \left|\eta_{i}\right|<5,
\end{array}
$$

where $\Delta R=\sqrt{\Delta \Phi^{2}+\Delta \eta^{2}}$ is the separation in the rapidityazimuth plane, $p_{T}^{\gamma, \ell, b, j}$ are the transverse momentum of photons, leptons, $b$-jets and jets.

In Fig. 3, we plot some differential distributions for signals and SM backgrounds at the HE-LHC, such as the transverse momentum distributions of the two photons, the separations $\Delta R_{\gamma \gamma}$ and $\Delta R_{\gamma \ell}$, the invariant mass distributions of the two photons, $M_{\gamma_{1} \gamma_{2}}$, and the transverse mass distribution for the top quark. Here the transverse masses of the top quark are defined as [52]

$$
\begin{aligned}
M_{T}^{2}(b \ell) \equiv & \left(\sqrt{\left(p_{\ell}+p_{b}\right)^{2}+\left|\vec{p}_{T, \ell}+\vec{p}_{T, b}\right|^{2}}+\left|\vec{p}_{T}\right|\right)^{2} \\
& -\left|\vec{p}_{T, \ell}+\vec{p}_{T, b}+\vec{p}_{T}\right|^{2},
\end{aligned}
$$

where $\vec{p}_{T, \ell}$ and $\vec{p}_{T, b}$ are the transverse momentums of the charged leptons and $b$-quark, respectively, and $\vec{p}_{T}$ is the miss- ing transverse momentum determined by the negative sum of visible momenta in the transverse direction.

One can see that the two photons in the signal and the resonant backgrounds have the harder $p_{T}$ spectrum than those in the non-resonant backgrounds. Furthermore, the signal and the resonant backgrounds have the diphoton invariant-mass peak at the mass of Higgs boson. Besides, the cut on the transverse mass of the top candidate is needed since the lepton and $b$-jet are assumed to originate from the leptonic decaying top quark.

According to the above analysis, we can impose the following set of cuts.

- Cut 1: There are only one isolated lepton, especially one positive charge lepton is required for $u g \rightarrow t h$ process, The leading $b$-tagged jet is required to have $p_{T}>30$ $\mathrm{GeV}$. Furthermore, an event is rejected if the $p_{T}$ of the subleading jet or subleading $b$-tagged jet is greater than $30 \mathrm{GeV}$.

- Cut 2: At least two photons and the leading (sub-leading) photons with $p_{T}>70$ (40) GeV, $\Delta R_{\gamma_{1}, \gamma_{2}}<1.8$ and $\Delta R_{\gamma_{1}, \ell}>2.0$.

- Cut 3: The invariant mass of the two diphotons is required as $\left|M_{\gamma_{1} \gamma_{2}}-m_{h}\right|<5 \mathrm{GeV}$.

- Cut 4: The transverse mass of the $W$ candidate is required as $M_{T}^{W}>30 \mathrm{GeV}^{1}$, and the transverse mass of the top quark is required as $100 \mathrm{GeV}<M_{T}^{b \ell}<180 \mathrm{GeV}$.

We use the same selection cuts for the FCC-hh analysis at $100 \mathrm{TeV}$ because the distributions are very similar to the case of HE-LHC. The effects of the suitable cuts on the signal and SM background processes are illustrated in Tables 2, 3. One can see that, the jet veto in cut 1 significantly reduce the backgrounds which contain two $b$ jets from the decay of the top quarks. The cut on the invariant-mass of the mass of Higgs boson can reduce the non-resonance backgrounds largely. At the end of the cut flow, the total SM backgrounds are very small, and there are 90 events at the FCC-hh with the integrated luminosity of $30 \mathrm{fb}^{-1}$. Furthermore, the cross section for the $u g \rightarrow t h$ process is more than twice that of the $p p \rightarrow t \bar{t} \rightarrow t h j$ process, while the cross section for the $c g \rightarrow t h$ process is smaller than that of the $p p \rightarrow t \bar{t} \rightarrow$ $t h j$ process. Thus we can not neglect the contribution from the top pair production with the FCNC decay process when considering the sensitivities on $\kappa_{t c h}$.

\footnotetext{
${ }^{1}$ Due to existence of neutrino in the final state of signal events, the $W$ candidate transverse mass is reconstructed as $M_{T}^{W}=$ $\sqrt{2 p_{T, \ell} E_{T}^{\text {miss }}-\vec{p}_{T, \ell} \cdot \vec{E}_{T}^{\text {miss }}}$.
} 

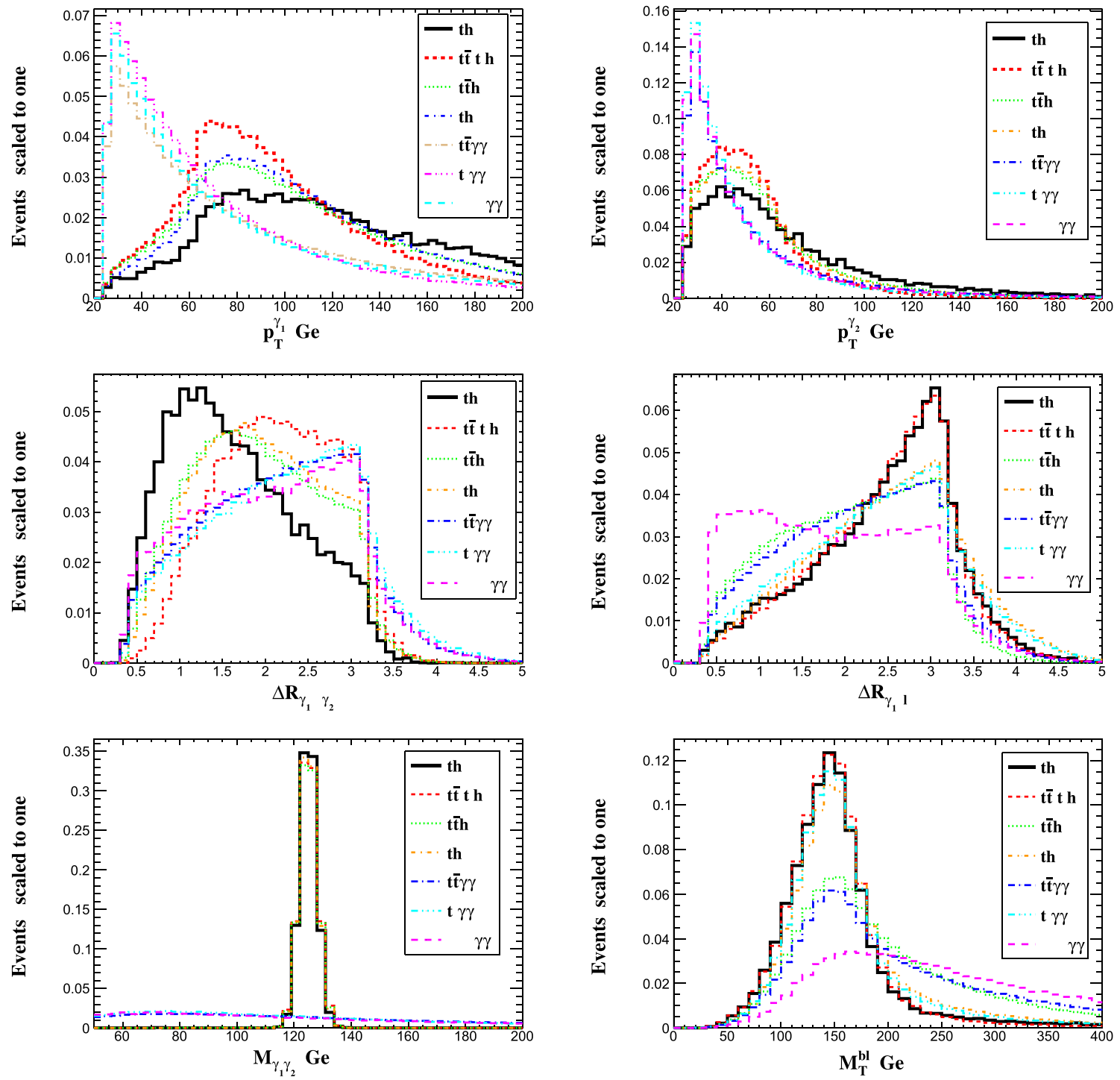

Fig. 3 Normalised distributions for the signals and SM backgrounds at the HE-LHC

Table 2 The cut flow of the cross sections (in ab) for the signals and SM backgrounds at the HE-LHC with $\kappa_{t u h}=0.04$ and $\kappa_{t c h}=0.04$ (in the bracket)

\begin{tabular}{|c|c|c|c|c|c|c|c|}
\hline \multirow[t]{2}{*}{ Cuts } & \multicolumn{2}{|l|}{ Signal } & \multicolumn{5}{|c|}{ Backgrounds } \\
\hline & $p p \rightarrow t h$ & $\overline{t \bar{t} \rightarrow t h j}$ & $\overline{t \bar{t} h}$ & $t h j$ & $t \bar{t} \gamma \gamma$ & $t j \gamma \gamma$ & $W^{ \pm} \gamma \gamma j j$ \\
\hline Basic cuts & $77(25)$ & 344 (324) & 254 & 32 & 3599 & 4148 & 4987 \\
\hline Cut 1 & $25(7)$ & $25(36)$ & $2.7(2.7)$ & $1.3(1.2)$ & $36(37)$ & $326(183)$ & $2152(235)$ \\
\hline Cut 2 & $9.9(2.4)$ & $4.3(6.5)$ & $0.46(0.49)$ & $0.22(0.2)$ & $0.12(1.4)$ & $0.56(3.2)$ & $0.29(3.1)$ \\
\hline Cut 3 & $9.2(2.2)$ & $3.9(5.9)$ & $0.41(0.44)$ & $0.2(0.19)$ & $0.06(0.11)$ & $0.35(0.32)$ & $0.26(0.22)$ \\
\hline Cut 4 & $5.8(1.4)$ & $2.5(3.9)$ & $0.1(0.1)$ & $0.1(0.1)$ & $0.02(0.03)$ & $0.2(0.1)$ & $0.07(0.07)$ \\
\hline
\end{tabular}

\subsection{Sensitivity at the HE-LHC and FCC-hh}

To estimate the expected discovery and exclusion significance, $Z_{\text {disc }}$ and $Z_{\text {excl }}$, for the small number of events, we use the following expression [53]:

$$
\begin{aligned}
& Z_{\mathrm{disc}}=\sqrt{2\left[(s+b) \ln \left(\frac{(s+b)\left(1+\delta^{2} b\right)}{b+\delta^{2} b(s+b)}\right)-\frac{1}{\delta^{2}} \ln \left(1+\delta^{2} \frac{s}{1+\delta^{2} b}\right)\right]} \\
& Z_{\mathrm{excl}} \\
& =\sqrt{2\left[s-b \ln \left(\frac{b+s+x}{2 b}\right)-\frac{1}{\delta^{2}} \ln \left(\frac{b-s+x}{2 b}\right)\right]-(b+s-x)\left(1+\frac{1}{\delta^{2} b}\right)},
\end{aligned}
$$


Table 3 The cut flow of the cross sections (in ab) for the signals and SM backgrounds at the FCC-hh with $\kappa_{t u h}=0.04$ and $\kappa_{t c h}=0.04$ (in the bracket)

\begin{tabular}{|c|c|c|c|c|c|c|c|}
\hline \multirow[t]{2}{*}{ Cuts } & \multicolumn{2}{|l|}{ Signal } & \multicolumn{5}{|c|}{ Backgrounds } \\
\hline & $p p \rightarrow t h$ & $t \bar{t} \rightarrow t h j$ & $t \bar{t} h$ & $t h j$ & $t \bar{t} \gamma \gamma$ & $t j \gamma \gamma$ & $W^{ \pm} \gamma \gamma j j$ \\
\hline Basic cuts & $683(415)$ & $4490(4381)$ & 7657 & 592 & 72324 & 61740 & 55158 \\
\hline Cut 1 & $113(73)$ & $96(278)$ & $17(36)$ & $11(19)$ & 159 (319) & 1109 (2129) & $423(822)$ \\
\hline Cut 2 & $41(24)$ & $16(43)$ & $2.7(6.4)$ & $1.7(1.1)$ & $6(3.3)$ & $18(3.3)$ & $17(3.3)$ \\
\hline Cut 3 & $40(23)$ & $15.8(42)$ & $2.6(6.1)$ & $1.6(2.9)$ & $0.4(0.65)$ & $1.3(2.3)$ & $1.3(2.5)$ \\
\hline Cut 4 & $28(15.5)$ & $10.5(25.8)$ & $0.7(1.5)$ & $1(1.8)$ & $0.08(0.08)$ & $0.9(1.6)$ & $0.3(0.4)$ \\
\hline
\end{tabular}

Table 4 The upper limits on BR $(t \rightarrow q h)$ at 95\% CL obtained at the HE-LHC and FCC-hh. We consider systematic errors of $0 \%$, 10\% and 20\% on the SM background events

\begin{tabular}{|c|c|c|c|c|c|c|}
\hline \multirow[t]{2}{*}{ Branching fraction } & \multicolumn{3}{|c|}{ HE-LHC, $15 \mathrm{ab}^{-1}$} & \multicolumn{3}{|c|}{ FCC-hh, $30 \mathrm{ab}^{-1}$} \\
\hline & $\delta=0$ & $\delta=10 \%$ & $\delta=20 \%$ & $\delta=0$ & $\delta=10 \%$ & $\delta=20 \%$ \\
\hline $\mathrm{BR}(t \rightarrow u h)$ & $4.4 \times 10^{-5}$ & $4.5 \times 10^{-5}$ & $4.8 \times 10^{-5}$ & $1.3 \times 10^{-5}$ & $1.7 \times 10^{-5}$ & $2.4 \times 10^{-5}$ \\
\hline $\mathrm{BR}(t \rightarrow c h)$ & $6.4 \times 10^{-5}$ & $6.5 \times 10^{-5}$ & $6.9 \times 10^{-5}$ & $1.6 \times 10^{-5}$ & $2.5 \times 10^{-5}$ & $3.9 \times 10^{-5}$ \\
\hline
\end{tabular}
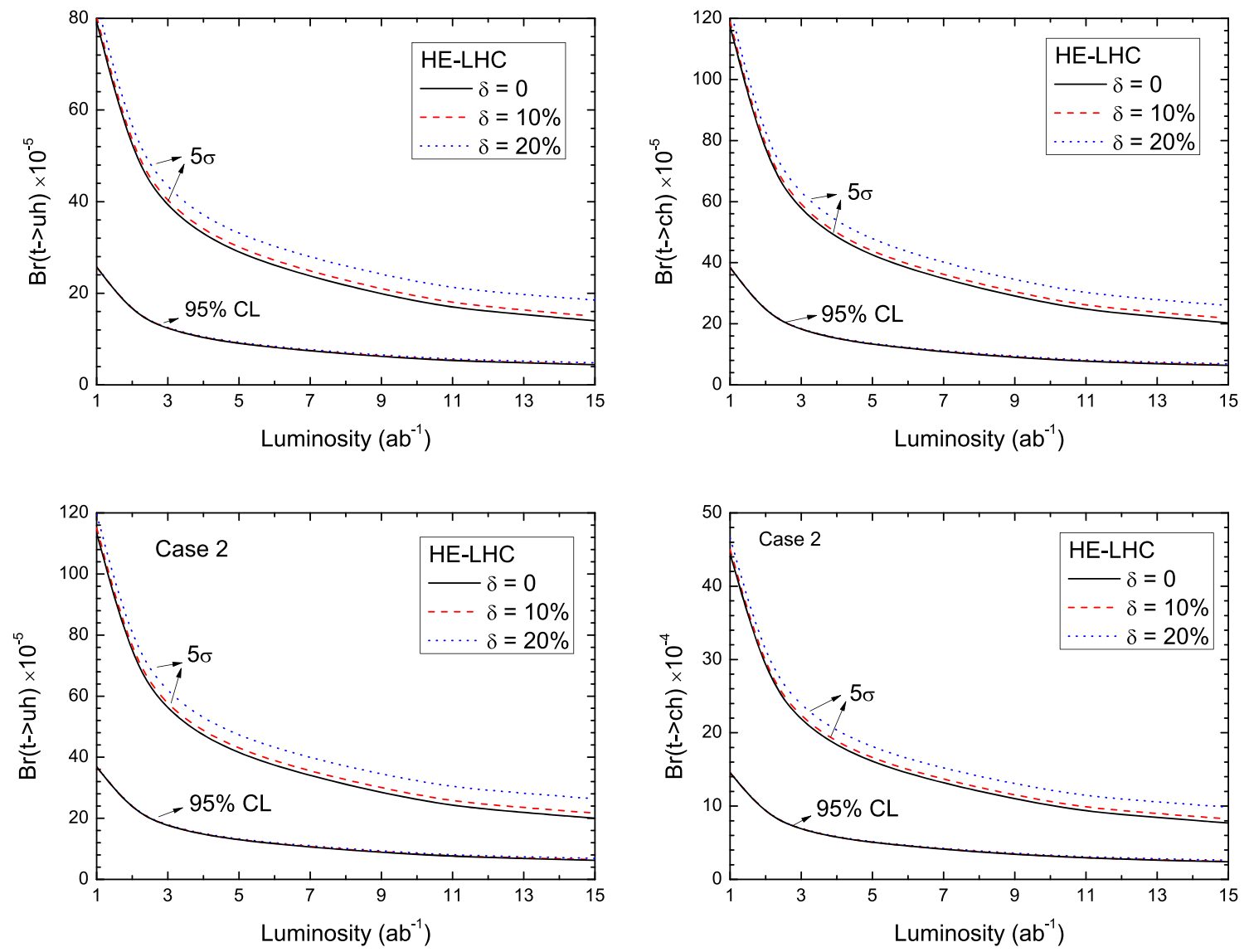

Fig. 4 The $95 \%$ and $5 \sigma$ contour plots for the signal in $L_{i n t}-B r(t \rightarrow u h)$ (left) and $L_{i n t}-B r(t \rightarrow c h)$ (right) planes at HE-LHC. For comparison, the results also plot the under two figures for the case of only considering the signal from the $p p \rightarrow t h$ process

with

$x=\sqrt{(s+b)^{2}-4 \delta^{2} s b^{2} /\left(1+\delta^{2} b\right)}$.
Here, $s$ and $b$ represent the total signal and SM background events, respectively. $\delta$ is the percentage systematic error on the SM background estimate. In this work we choose three cases: $\delta=0 \%, 10 \%, 20 \%$. In the limit of $\delta \rightarrow 0$, the above 

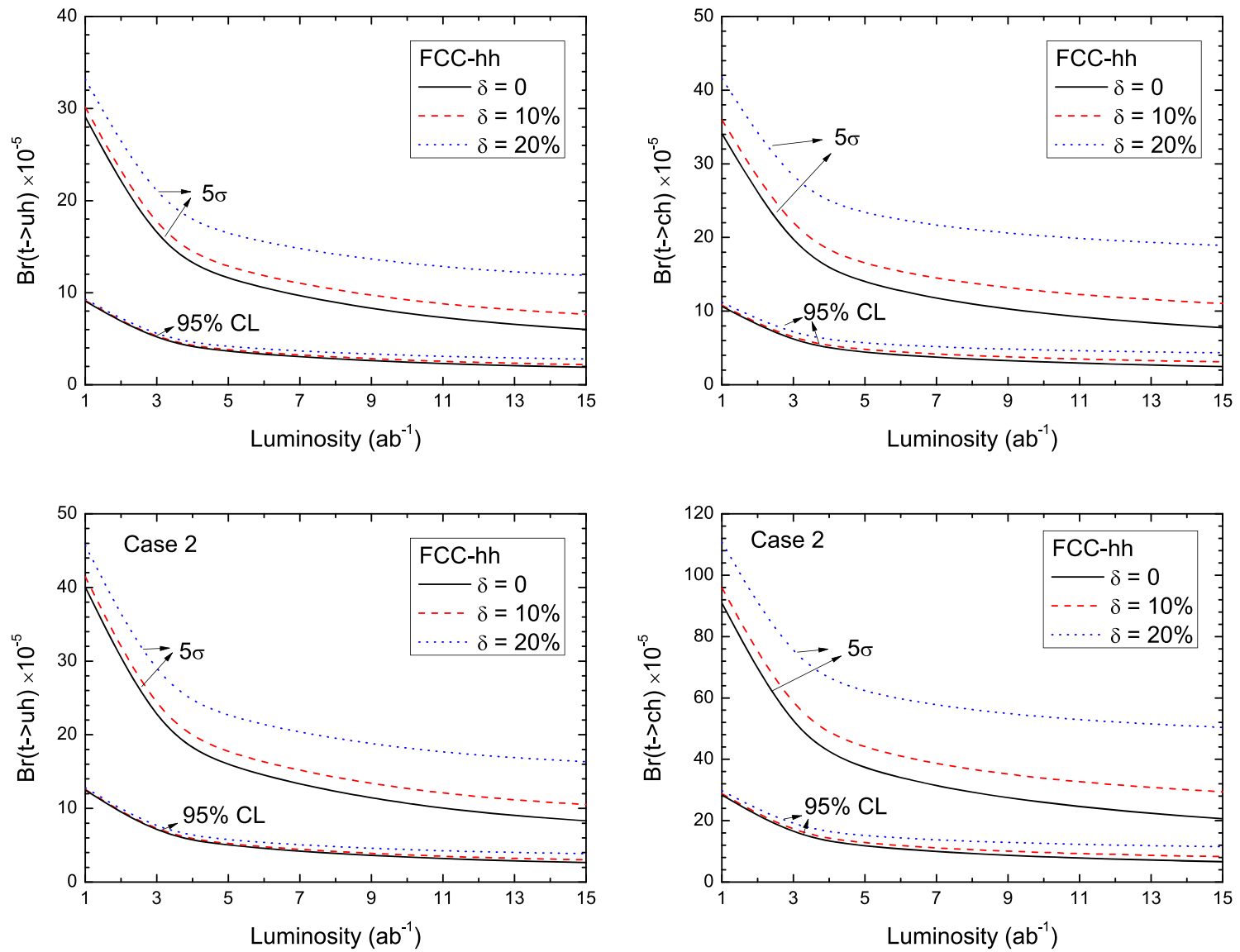

Fig. 5 Same for Fig. 4 but at the FCC-hh

expressions can be simplified as

$$
\begin{aligned}
& Z_{\text {disc }}=\sqrt{2[(s+b) \ln (1+s / b)-s]}, \\
& Z_{\text {excl }}=\sqrt{2[s-b \ln (1+s / b)]}
\end{aligned}
$$

We define the regions with $Z_{\text {excl }} \leq 1.645$ as those that can be excluded at $95 \% \mathrm{CL}$, and $Z_{\mathrm{disc}} \geq 5$ as discovered regions [53].

In Table 4, we list the exclusion limits at 95\% CL at the future HE-LHC and FCC-hh, respectively, with three systematic error cases: $\delta=0 \%, \delta=10 \%$ and $\delta=20 \%$. One can see that, the sensitivities are slightly weaker than those without any systematic error, being of the order of $10^{-5}$ at the 95\% CL both at the HE-LHC and FCC-hh.

In Figs. 4, 5, the 95\% CL and 5 $\sigma$ lines are drawn as a function of the integrated luminosity and $B R(t \rightarrow q h)$. We also plot the results for the case of only considering the signal from the process $p p \rightarrow t h$ (marked as Case 2 in the figures). For the case of $\delta=10 \%$, one can see that the $5 \sigma$ discovery sensitivity of $\operatorname{Br}(t \rightarrow q h)(q=u / c)$ is about $1.5 \times 10^{-4}\left(2.2 \times 10^{-4}\right)$ at the HE-LHC with the integrated luminosity is $15 \mathrm{ab}^{-1}$, and about $6 \times 10^{-5}\left(9 \times 10^{-5}\right)$ at the

FCC-hh with the integrated luminosity is $30 \mathrm{ab}^{-1}$, respectively. However, the numerical results are changed significantly for the sensitivity of $\operatorname{Br}(t \rightarrow c h)$ in Case 2. This is mainly because the cross sections of the $u g \rightarrow t h$ process and $p p \rightarrow t \bar{t} \rightarrow t h j$ process are much larger than that of the $c g \rightarrow$ th process, which has been discussed earlier. For example, at the HE-LHC with the integrated luminosity is $15 \mathrm{ab}^{-1}$ and the FCC-hh with the integrated luminosity is $30 \mathrm{ab}^{-1}$, the upper limits at $95 \% \mathrm{CL}$ on $\mathrm{Br}(t \rightarrow c h)$ with $\delta=10 \%$ are changed as $2.4 \times 10^{-4}$ and $6.6 \times 10^{-5}$, respectively, and the $5 \sigma$ CL discovery sensitivity changed as $8.3 \times 10^{-4}$ and $2.4 \times 10^{-4}$, respectively.

Very recently, many phenomenological studies available in literature have extensively investigated the tqh FCNC couplings via the top pair production process at the future hadron colliders, see e.g. Refs. [28,54-56] for the most recent reviews. It is worth comparing the limits obtained in this study with those obtained in other groups, which are summarized in Table 5. One can see that the limits on the branching ratios are expected to be of $\mathcal{O}\left(10^{-5}\right)$. Therefore, we expect to provide the complementary information for detecting such anomalous couplings at the future HE-LHC and FCC-hh. 
Table 5 The recent projected 95\% CL limits on $\operatorname{Br}(t \rightarrow q h)$ from different channels at the HE-LHC and FCC-hh

\begin{tabular}{|c|c|c|}
\hline Channels & Data Set & 95\% CL Limits \\
\hline \multirow[t]{4}{*}{$t \bar{t} \rightarrow W(\rightarrow \ell v) b+h(\rightarrow \gamma \gamma) q$} & HE-LHC, $15 \mathrm{ab}^{-1} @ 27 \mathrm{TeV}$ & $B r(t \rightarrow u h)<7.0 \times 10^{-5}$ \\
\hline & & $B r(t \rightarrow c h)<8.5 \times 10^{-5}$ \\
\hline & FCC-hh, $30 \mathrm{ab}^{-1} @ 100 \mathrm{TeV}$ & $B r(t \rightarrow u h)<2.3 \times 10^{-5}[28]$ \\
\hline & & $B r(t \rightarrow c h)<3.0 \times 10^{-5}[28]$ \\
\hline \multirow[t]{2}{*}{$t \bar{t} \rightarrow W(\rightarrow \ell v) b+h\left(\rightarrow W W^{*}\right) q$} & HE-LHC, $3 \mathrm{ab}^{-1} @ 27 \mathrm{TeV}$ & $B r(t \rightarrow c h)<6.1 \times 10^{-4}$ \\
\hline & FCC-hh, 3 ab $^{-1} @ 100 \mathrm{TeV}$ & $B r(t \rightarrow c h)<2.0 \times 10^{-4}$ \\
\hline$t \bar{t} \rightarrow W b+h(\rightarrow \gamma \gamma) q$ & FCC-hh, $10 \mathrm{ab}^{-1} @ 100 \mathrm{TeV}$ & $B r(t \rightarrow c h)<2.8 \times 10^{-5}$ \\
\hline \multirow[t]{4}{*}{$p p \rightarrow t t \bar{t}(\bar{t} t \bar{t})$} & HE-LHC, $15 \mathrm{ab}^{-1} @ 27 \mathrm{TeV}$ & $B r(t \rightarrow u h)<7.6 \times 10^{-5}[56]$ \\
\hline & & $B r(t \rightarrow c h)<7.0 \times 10^{-4}$ \\
\hline & FCC-hh, $10 \mathrm{ab}^{-1} @ 100 \mathrm{TeV}$ & $B r(t \rightarrow u h)<4.8 \times 10^{-5}$ \\
\hline & & $B r(t \rightarrow c h)<9.7 \times 10^{-5}$ \\
\hline
\end{tabular}

\section{Conclusion}

In this work, we have studied the top-Higgs FCNC interactions, i.e., $\operatorname{tgh}(q=u, c)$, at the HE-LHC and FCC-hh by performing a full simulation for the process: $p p \rightarrow t h$ with the top leptonic decay channel and $h \rightarrow \gamma \gamma$ decay channel. In the case the SM background is known with negligible uncertainty, our results show that $95 \%$ CL limits on the branching ratios of $t \rightarrow u(c) h$, have been found to be $4.4(6.4) \times 10^{-5}$ at the HE-LHC with an integrated luminosity of $15 \mathrm{ab}^{-1}$ and $1.3(1.6) \times 10^{-5}$ at the FCC-hh with an integrated luminosity of $30 \mathrm{ab}^{-1}$. Meanwhile, the $5 \sigma$ discovery sensitivity of $\operatorname{Br}(t \rightarrow u(c) h)$ is about $1.4 \times 10^{-4}\left(2.0 \times 10^{-4}\right)$ at the HELHC, and about $4.1 \times 10^{-5}\left(5 \times 10^{-5}\right)$ at the FCC-hh, respectively. Therefore, we expect to provide the complementary information for detecting such anomalous couplings at the future HE-LHC and FCC-hh.

Acknowledgements We would like thank Y.-B Liu for useful discussion. This work is supported by the Foundation of He'nan Educational Committee (Grant no. 2015GGJS-059).

Data Availability Statement This manuscript has associated data in a data repository. [Authors' comment: My manuscript has no associated data.]

Open Access This article is licensed under a Creative Commons Attribution 4.0 International License, which permits use, sharing, adaptation, distribution and reproduction in any medium or format, as long as you give appropriate credit to the original author(s) and the source, provide a link to the Creative Commons licence, and indicate if changes were made. The images or other third party material in this article are included in the article's Creative Commons licence, unless indicated otherwise in a credit line to the material. If material is not included in the article's Creative Commons licence and your intended use is not permitted by statutory regulation or exceeds the permitted use, you will need to obtain permission directly from the copyright holder. To view a copy of this licence, visit http://creativecomm ons.org/licenses/by/4.0/.

Funded by SCOAP ${ }^{3}$.

\section{References}

1. G. Aad et al., ATLAS Collaboration. Phys. Lett. B 716, 1 (2012)

2. V. Khachatryan et al., CMS Collaboration. Phys. Lett. B 716, 30 (2012)

3. J.A. Aguilar-Saavedra, Acta Phys. Polon. B 35, 2695 (2004)

4. J.A. Aguilar-Saavedra, Nucl. Phys. B 821, 215 (2009)

5. J.L. Diaz-Cruz, H.J. He, C.P. Yuan, Phys. Lett. B 530, 179 (2002)

6. J. Cao, C. Han, L. Wu, J.M. Yang, M. Zhang, Eur. Phys. J. C 74, 3058 (2014)

7. T.J. Gao, T.F. Feng, F. Sun, H.B. Zhang, S.M. Zhao, Chin. Phys. C 39, 073101 (2015)

8. C. Kao, H.Y. Cheng, W.S. Hou, J. Sayre, Phys. Lett. B 716, 225 (2012)

9. T. Han, R. Ruiz, Phys. Rev. D 89, 074045 (2014)

10. G. Abbas, A. Celis, X.Q. Li, J. Lu, A. Pich, JHEP 1506, 005 (2015)

11. F.J. Botella, G.C. Branco, M. Nebot, M.N. Rebelo, Eur. Phys. J. C 76, 161 (2016)

12. M.A. Arroyo-Ureña, J.L. Diaz-Cruz, E. Díaz, J.A. Orduz-Ducuara, Chin. Phys. C 40, 123103 (2016)

13. D.T. Huong, N.T. Duy, Eur. Phys. J. C 80, 439 (2020)

14. H.J. He, T.M.P. Tait, C.P. Yuan, Phys. Rev. D 62, 011702 (2000)

15. M. Badziak, K. Harigaya, Phys. Rev. Lett. 120, 211803 (2018)

16. T.M.P. Tait, C.-P. Yuan, Phys. Rev. D 63, 014018 (2000)

17. J.A. Aguilar-Saavedra, G.C. Branco, Phys. Lett. B 495, 347 (2000)

18. Q.H. Cao, J. Wudka, C.-P. Yuan, Phys. Lett. B 658, 50 (2007)

19. M. Aaboud et al., ATLAS Collaboration. JHEP 1710, 129 (2017)

20. M. Aaboud et al., ATLAS Collaboration. Phys. Rev. D 98, 032002 (2018)

21. M. Aaboud et al., ATLAS Collaboration. JHEP 1905, 123 (2019)

22. A.M. Sirunyan et al., CMS Collaboration. JHEP 1806, 102 (2018)

23. ATLAS Collaboration, ATL-PHYS-PUB-2016-019

24. ATLAS Collaboration, ATL-PHYS-PUB-2013-012

25. P. Mandrik [FCC study Group], J. Phys. Conf. Ser. 1390 (2019) 012044

26. M. Benedikt, F. Zimmermann, Nucl. Instrum. Meth. A 907, 200 (2018)

27. N. Arkani-Hamed, T. Han, M.L. Mangano, L.-T. Wang, Phys. Rep. 652, 1 (2016)

28. Y.-B. Liu, S. Moretti, Phys. Rev. D 101, 075029 (2020)

29. Y. Wang, F.P. Huang, C.S. Li, B.H. Li, D.Y. Shao, J. Wang, Phys. Rev. D 86, 094014 (2012)

30. X. Chen, L. Xia, Phys. Rev. D 93, 113010 (2016)

31. S. Khatibi, M.M. Najafabadi, Phys. Rev. D 89, 054011 (2014)

32. D. Atwood, S.K. Gupta, A. Soni, JHEP 1410, 057 (2014) 
33. Y.B. Liu, Z.J. Xiao, Phys. Lett. B 763, 458 (2016)

34. A. Greljo, J.F. Kamenik, J. Kopp, JHEP 1407, 046 (2014)

35. Y.B. Liu, Z.J. Xiao, Phys. Rev. D 94, 054018 (2016)

36. L. Wu, JHEP 1502, 061 (2015)

37. Y.B. Liu, S. Moretti, Chin. Phys. C 43, 013102 (2019)

38. A. Alloul, N.D. Christensen, C. Degrande, C. Duhr, B. Fuks, Comput. Phys. Commun. 185, 2250 (2014)

39. C. Degrande, C. Duhr, B. Fuks, D. Grellscheid, O. Mattelaer, T. Reiter, Comput. Phys. Commun. 183, 1201 (2012)

40. J. Alwall, R. Frederix, S. Frixione, V. Hirschi, F. Maltoni, O. Mattelaer, H.-S. Shao, T. Stelzer, P. Torrielli, M. Zaro, JHEP 1407, 079 (2014)

41. R.D. Ball et al., NNPDF Collaboration. JHEP 1504, 040 (2015)

42. M. Tanabashi et al., Particle Data Group. Phys. Rev. D 98, 030001 (2018)

43. T. Sjöstrand, S. Ask, J.R. Christiansen et al., Comput. Phys. Commun. 191, 159 (2015)

44. M. Cacciari, G.P. Salam, G. Soyez, Eur. Phys. J. C 72, 1896 (2012)

45. M. Cacciari, G.P. Salam, G. Soyez, JHEP 0804, 063 (2008)

46. J. de Favereau et al., DELPHES 3 Collaboration. JHEP 1402, 057 (2014)
47. E. Conte, B. Fuks, G. Serret, Comput. Phys. Commun. 184, 222 (2013)

48. C. Zhang, F. Maltoni, Phys. Rev. D 88, 054005 (2013)

49. M. L. Mangano et al., CERN Yellow Rep. (2017) no3. 1-254, arXiv:1607.01831 [hep-ph]

50. M. Cepeda et al., CERN Yellow Rep. Monogr. 7, 221 (2019). arXiv:1902.00134 [hep-ph]

51. Y. Zhang, W.G. Ma, R.Y. Zhang, C. Chen, L. Guo, Phys. Lett. B 738, 1 (2014)

52. E. Conte, B. Dumont, B. Fuks, C. Wymant, Eur. Phys. J. C 74, 3103 (2014)

53. G. Cowan, K. Cranmer, E. Gross and O. Vitells, Eur. Phys. J. C 71 (2011) 1554, Erratum: [Eur. Phys. J. C 73 (2013) 2501]

54. R. Jain, C. Kao, Phys. Rev. D 99, 055036 (2019)

55. A. Papaefstathiou, G. Tetlalmatzi-Xolocotzi, Eur. Phys. J. C 78, $214(2018)$

56. H. Khanpour, Nucl. Phys. B 958, 115141 (2020). arXiv:1909.03998 [hep-ph] 